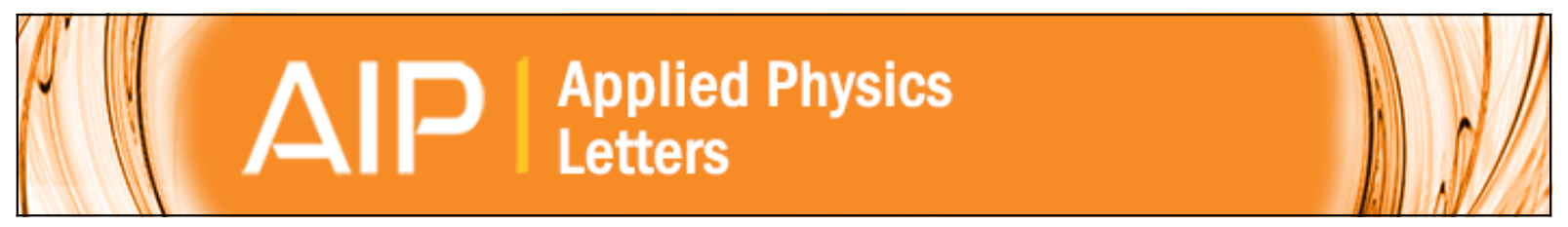

\title{
Nanoscopic electric potential probing: Influence of probe-sample interface on spatial resolution
}

S. B. Kuntze, E. H. Sargent, St. J. Dixon-Warren, J. K. White, K. Hinzer, and D. Ban

Citation: Applied Physics Letters 84, 601 (2004); doi: 10.1063/1.1643534

View online: http://dx.doi.org/10.1063/1.1643534

View Table of Contents: http://scitation.aip.org/content/aip/journal/apl/84/4?ver=pdfcov

Published by the AIP Publishing

\section{Articles you may be interested in}

Time evolution studies of the electrostatic surface potential of low-temperature-grown GaAs using electrostatic force microscopy

J. Appl. Phys. 97, 043703 (2005); 10.1063/1.1844615

Spectroscopic method of strain analysis in semiconductor quantum-well devices

J. Appl. Phys. 96, 4056 (2004); 10.1063/1.1791754

Influence of the electrical contact on the reliability of InP-based ridge waveguide distributed feedback semiconductor diode lasers for telecommunications applications

J. Vac. Sci. Technol. A 22, 803 (2004); 10.1116/1.1701855

Distribution of built-in electrical potential in GalnP 2 / GaAs tandem-junction solar cells Appl. Phys. Lett. 83, 1572 (2003); 10.1063/1.1602575

Measurement of built-in electrical potential in III-V solar cells by scanning Kelvin probe microscopy J. Appl. Phys. 93, 10035 (2003); 10.1063/1.1573736

\section{AIP $\mid$ APL Photonics \\ APL Photonics is pleased to announce Benjamin Eggleton as its Editor-in-Chief}

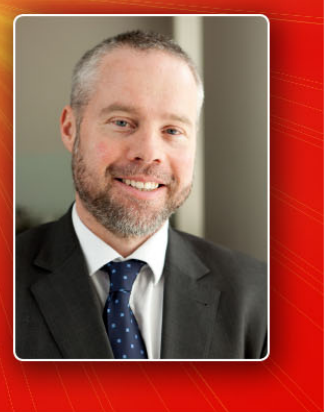




\title{
Nanoscopic electric potential probing: Influence of probe-sample interface on spatial resolution
}

\author{
S. B. Kuntze and E. H. Sargent ${ }^{\mathrm{a})}$ \\ Edward S. Rogers Sr. Department of Electrical and Computer Engineering, University of Toronto, 10 King's \\ College Road, Toronto, Ontario, Canada M5S $3 G 4$ \\ St. J. Dixon-Warren, J. K. White, and K. Hinzer \\ Bookham Technology plc, 1-10 Brewer Hunt Way, Kanata, Ontario, Canada K2K 2B5 \\ D. Ban \\ Institute for Microstructural Sciences, National Research Council, 1200 Montreal Road, Ottawa, Ontario, \\ Canada K1A 0R6
}

(Received 19 June 2003; accepted 1 December 2003)

\begin{abstract}
Electric potential probing on the nanometer scale elucidates the operation of actively driven conducting, semiconducting, insulating and semi-insulating devices and systems. Spatial resolution of this analysis technique is shown to depend on the time required for the voltage measurement circuit to reach steady state with the local electric potential of the sample. Scanning voltage microscopy on actively biased buried heterostructure lasers reveals this time to be intrinsically long $\left(10^{-2} \mathrm{~s}\right.$ to $1 \mathrm{~s}$ ) and to depend on material doping type ( $n$ - or $p$-type) and scan direction (to increasing or decreasing sample potential). The bandstructure of the probe-sample interface is examined and is shown to provide high incremental contact resistance to an equivalent circuit model of the measurement circuit. Practical scan speed limits are defined for accurate scanning electric potential measurements given a desired spatial resolution. (c) 2004 American Institute of Physics.
\end{abstract} [DOI: $10.1063 / 1.1643534]$

Nanoscopic probing of the electric potential elucidates the operation of actively driven conducting, semiconducting, insulating, and semi-insulating devices and systems. In particular, scanning voltage microscopy (SVM) directly reveals the potential profiles of actively biased devices on the nanometer scale by combining high-impedance potentiometry with the spatial profiling of atomic force microscopy (AFM). SVM has offered substantial insights into the potential profiles that govern and limit device performance of actively biased complementary metal-oxide semiconductor transistors $^{1,2}$ and semiconductor lasers. ${ }^{3,4}$ Previously, potential profiles of active devices were available only through theoretical modeling and simulation, or considerably less directly through complex derivation from the results of scanning capacitance microscopy, ${ }^{5}$ scanning force microscopy, ${ }^{6}$ and Kelvin probe force microscopy. ${ }^{7}$

During earlier work, ${ }^{8}$ qualitative differences in twodimensional SVM potential maps were noted. These differences depended on whether the probe was scanned in the direction of increasing or decreasing sample potential. In the direction that tracked increasing sample potential, abrupt interfaces between adjacent material layers were more clearly delineated than those in the decreasing-potential scan direction. Images taken in decreasing-potential direction were notably more smeared, particularly over $p$-type InP.

In this letter, we examine the apparent differences in captured SVM images-whether captured in the increasing or decreasing potential direction on $n$ - or $p$-type semiconductors - and identify the energy band alignment at the probe-sample interface as the source of the observed

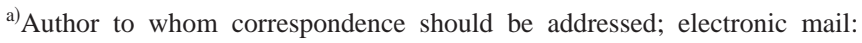
ted.sargent@utoronto.ca
}

differences. We show how spatial resolution is affected directly by the time needed for the measurement circuit to reach steady state with the sample and we quantify the associated time constants. The measurement circuit system (including the probe-sample interface) is considered as a resistance capacitance (RC) equivalent circuit with series resistance dependent on scan direction and material doping type due to the formation of a Schottky barrier at the interface. A limitation on the scan speed required for accurate SVM measurements is presented; the limit relationship is relevant to conductive scanning probe techniques having high series resistance.

Two experiments were conducted to investigate the material and directional dependencies so that the most accurate SVM data can be captured at the highest possible spatial resolution. In the first experiment, a conductive AFM tip was scanned over the $p-n-p-n$ current blocking layers of a dcbiased buried heterostructure $(\mathrm{BH})$ laser at various scan rates to observe hysteresis between the forward and reverse electric potential scans. In the second experiment, a $\mathrm{BH}$ laser was modulated with a square wave to quantify the time constants of the SVM measurement circuit on $n$ - and $p$-type InP. An atomic force microscope (Nanoscope III, Digital Instruments, Veeco Instruments, Woodbury, NY) was used in both experiments with conductive boron-doped diamond-coated tips (DDESP, Nanosensors, with tip radius $\sim 10 \mathrm{~nm}$ ) connected electrically to an ultrahigh-impedance voltmeter (6517A, Keithley, Cleveland, OH). Tip-sample forces were sufficient to penetrate the native InP oxide and to provide good electrical contact at the interface. The output of the initial preamplifier of the voltmeter (voltage follower) was connected directly to the AFM data capture port. The samples studied were InGaAsP/InP BH lasers with uncoated 


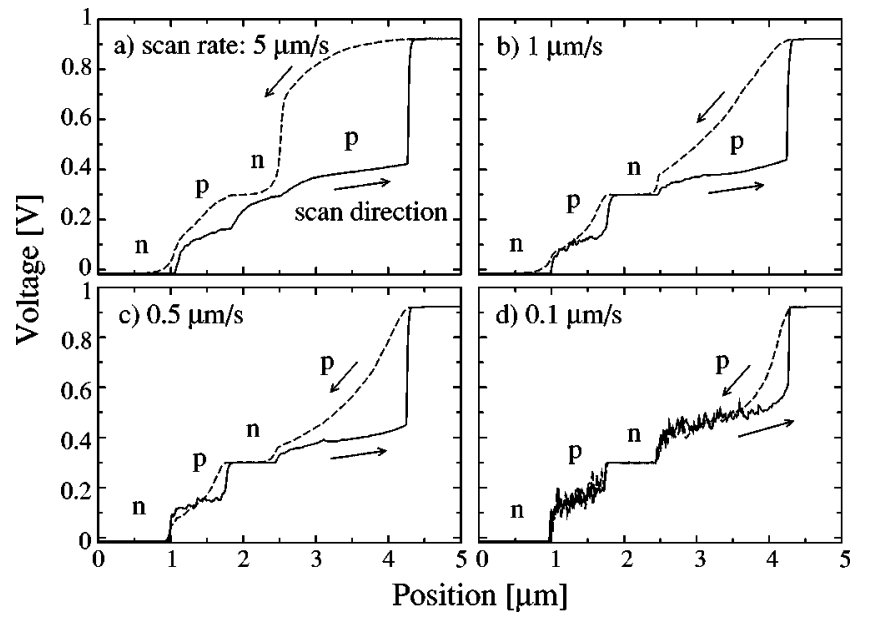

FIG. 1. Electric potential cross sections of the $p-n-p-n$ blocking layers of a forward-biased $\mathrm{BH}$ laser (arrows show spatial scan direction of probe). Significant hysteresis is observed between increasing (left- to right-hand side) and decreasing (right- to left-hand side) electric potential scans on $p$-type material at faster scan rates (a) $0.5 \mathrm{~Hz}$, (b) $0.1 \mathrm{~Hz}$, and (c) $0.05 \mathrm{~Hz}$. At the slowest scan rate of $0.01 \mathrm{~Hz}$ (d) steady state has been reached and the hysteresis is reduced acceptably; noise is observed at this speed since otherwise dominant probe-sample steady-state-approaching currents have subsided.

facets and metalized ohmic contacts. ${ }^{8}$ Nominal doping concentration of the $p-n-p-n$ current blocking layers was $10^{18} \mathrm{~cm}^{-3}$. The lasers were mounted on heat sinking carriers and were oriented to expose the facets to the AFM tip. Current-voltage $(I-V)$ characteristics of the lasers were recorded before and after the experiments to ensure normal device operation.

One-dimensional SVM cross-sectional potential profiles were measured across $p-n-p-n$ current-blocking layers of the dc-biased BH laser. 512 samples were captured along $5 \mu \mathrm{m}$ scans giving approximately $10 \mathrm{~nm}$ resolution limited ideally by the tip radius. Direct comparison of potential profiles in Fig. 1 reveals hysteresis between the different directional scans: The forward scan (left- to right-hand side) closely tracks the abrupt increasing potential changes from layer to layer and is relatively invariant to scan speed whereas the reverse scan (right- to left-hand side) converges to abrupt decreasing potential changes only for the slowest scan speed shown $(0.1 \mu \mathrm{m} / \mathrm{s})$. Exponential time constants were estimated from these results and are summarized in Table $\mathrm{I}$ in the " $p-n-p-n$ " column. Time constants on $p$-type InP are an order of magnitude slower than those on $n$-type InP; on $p$-type InP, the average time constant for the decreasing potential scan direction is several times slower than that for the increasing potential scan direction. Noise becomes apparent only during the slowest scan [see Fig. 1(d)].

TABLE I. Summary of time constants measured for $p-n-p-n$ scan and time resolved experiments, and estimated from $\mathrm{RC}$ equivalent circuit for each doping type (nominal concentration $10^{18} \mathrm{~cm}^{18}$ ) and potential change (potential increasing or decreasing).

\begin{tabular}{|c|c|c|c|c|}
\hline Doping & Potential & $p-n-p-n(\mathrm{~s})$ & Square wave (s) & RC model (s) \\
\hline$n$-type & Increasing & $4 \times 10^{-2}$ & $10^{-2}$ & $10^{-2}$ \\
\hline$n$-type & Decreasing & $5 \times 10^{-2}$ & $10^{-2}$ & $10^{-2}$ \\
\hline$p$-type & Increasing & $3 \times 10^{-1}$ & $10^{-1}$ & $10^{-1}$ \\
\hline$p$-type & Decreasing & $9 \times 10^{-1}$ & $9 \times 19^{-1}$ & AlP content is \\
\hline
\end{tabular}

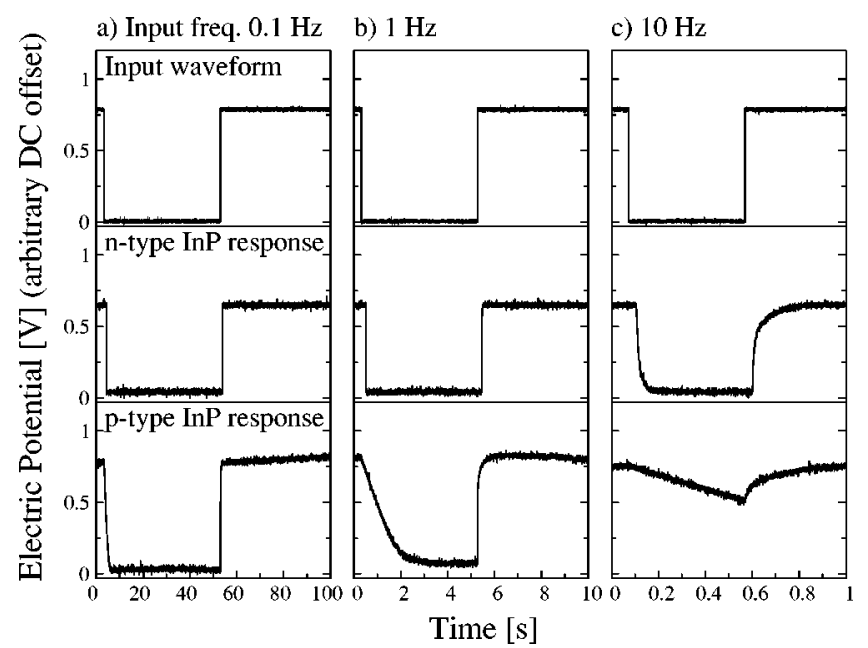

FIG. 2. Time resolution of the SVM measurement circuit on BH laser: Input wave form (top row), $n$-type response (middle row), and $p$-type response (bottom row), InP material $10^{18} \mathrm{~cm}^{-3}$ nominal doping, biased with square waves at (a) $0.1 \mathrm{~Hz}$, (b) $1 \mathrm{~Hz}$, and (c) $10 \mathrm{~Hz}$. Faster time response of $n$-type InP is observed. Average time constants are summarized in Table I.

To further examine the behavior of the tip-sample interface and measurement circuit, the SVM setup was modified for the second experiment: A 50\% duty-cycle square-wave bias was supplied to the $\mathrm{BH}$ laser and the potential output from the stationary AFM tip was captured on an oscilloscope via the voltmeter preamplifier output port. A similar setup was used by Trenkler et al. ${ }^{2}$ to characterize SVM probes. Figure 2 shows the time response for $n$ - and $p$-type InP of the $\mathrm{BH}$ laser for increasing bias frequency. The average time constants are summarized in Table I in the "Square wave" column. Again, time constants for $p$-type InP are an order of magnitude slower than those on $n$-type InP and tracking decreasing potential is several times slower than tracking increasing potential. At $10 \mathrm{~Hz}$ input bias [Fig. 2(c)], the $p$-type response shows the effects of severe low-pass filtering. The voltmeter was isolated and found to have a time response many orders of magnitude faster than those of the tipsample interface shown in Figs. 1 and 2.

The response shown in Fig. 2 is characteristic of a lowpass filter. There is no experimental evidence of high-pass filtering caused by junction and diffusion capacitances at the tip-sample interface, so we neglect these small-signal series capacitances. Previous scanning spreading resistance microscopy measurements with identical tips have shown ${ }^{9}$ that contact resistance dominates the series resistance of the diamond probes; ${ }^{10}$ contact resistances are on the order of $10^{8} \Omega$ for $n$-type InP and $10^{9}-10^{10} \Omega$ for $p$-type InP near steady state. The input impedance of the voltmeter consists of a very large resistance shunted with parasitic capacitance; the recorded SVM potential quantity is measured across this input impedance. Voltmeter input capacitance is fixed at 2 $\times 10^{-11} \mathrm{~F}$ and the input resistance is in excess of 2 $\times 10^{14} \Omega{ }^{11}$ Additional parasitic capacitance appears in shunt due to the electrical cables and connectors between the AFM tip and voltmeter and simply adds to the input capacitance to yield a total shunt capacitance on the order of magnitude of $10^{-10} \mathrm{~F}$. Since the input resistance of the voltmeter is several orders of magnitude greater than the series resistance of the interface, voltage division of the sample potential is insignificant, and the input resistance can be neglected. The 
a) Contact upon first probe tip placement or relocation

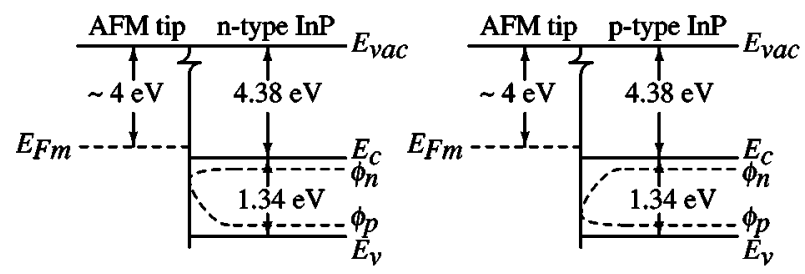

b) Steady-state contact after sufficient steady-state approach time

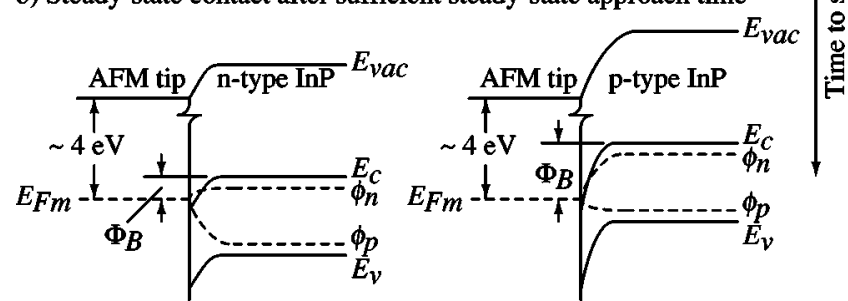

FIG. 3. Energy band diagram of tip-sample interface for $n$-type (left-hand side) and $p$-type (right-hand side) InP sample material: Tip Fermi level $E_{F m}$ (work function near $4 \mathrm{eV}$ ), conduction band $E_{c}$, valence band $E_{v}$, quasiFermi levels $\phi_{n}$ and $\phi_{p}$, and Schottky barrier height $\Phi_{B}$. (a) Nonsteadystate condition at first probe-sample contact or after probe relocation. (b) Steady-state results when the Fermi levels align with charge redistribution, causing band bending and formation of space-charge regions into the sample at the interface. Band bending is more severe for p-type InP and yields higher contact resistance.

equivalent circuit model is reduced to a single-time-constant $\mathrm{RC}$ circuit with series incremental contact resistance that varies with material doping type and scan direction, and a fixed parasitic shunt capacitance. Calculated RC time constants from this model are summarized in Table $\mathrm{I}$ in the "RC model" column and are consistent to the order of those measured in the experiments.

The source of the high contact resistance can be traced to the formation of a Schottky barrier at the tip-sample interface. The diamond grain coating of the AFM tip is doped sufficiently heavily with boron that it is appropriate to model the tip material as a metal ${ }^{12}$ with Fermi level $E_{F m}$ at the work function energy near $4 \mathrm{eV}$ below a vacuum. ${ }^{13,14}$ At the surface of the biased semiconductor laser, the quasi-Fermi levels $\phi_{n}$ and $\phi_{p}$ must converge as shown in Fig. 3(a) for both $n$ - and $p$-type InP. The electron affinity of $\mathrm{InP}$ is $4.38 \mathrm{eV}$ below a vacuum; the band-gap energy between the conduction-band edge $E_{c}$ and valence-band edge $E_{v}$ is 1.34 $\mathrm{eV}^{15}$ The process of approaching steady state at the tipsample interface takes place as charge is transferred between tip and sample to align the Fermi level of the tip and the convergence point of the quasi-Fermi levels of the sample. A depletion region arises in the sample as the pinned energy bands bend, leading to the formation of a Schottky barrier ${ }^{16}$ $\Phi_{B}$ as shown in Fig. 3(b). Contact resistance at the interface depends exponentially on the barrier height. ${ }^{16}$ Since the bandstructure of $p$-type InP gives rise to a larger barrier, the contact resistance must be substantially higher than that for $n$-type InP. Additionally, the greater depletion of carriers across the interface in the $p$-type case further slows the approach to steady state. Noise resulting from fluctuations in current at the interface appears at the slowest scan rate in Fig. 1(d) since the system has come to reasonable steady state and charge transfer is no longer dominated by Fermilevel alignment-induced current. Approaching steady state for decreasing sample potential is notably slower (particularly on $p$-type InP) since the barrier $\Phi_{B}$ and contact resistance increase for a lower convergence point of the quasiFermi levels, whereas approaching a higher steady-state potential decreases the barrier and contact resistance. With the parasitic capacitance of the measurement circuit fixed, the time-to-steady state varies only with this contact resistance.

To allow the probe-sample interface to reach steady state for each electric potential location at a desired spatial resolution $d$, the scan speed of the tip $v$ must limited such that $v<d / 5 \tau$, where $\tau$ is the longest time constant encountered on a given scan (according to material type and whether potential is increasing or decreasing); after five time constants, the tip is considered to be at steady steady with the sample. For example, at $v=0.5 \mu \mathrm{m} / \mathrm{s}$, roughly 50 samples/s can be captured accurately at a resolution of $10 \mathrm{~nm}$ on $n$-type InP since each sample has $0.05 \mathrm{~s}$ to reach steady state as shown in Fig. 1(c). The time required to reach tip-sample steady steady is shorter for scans in which increasing potential is measured so that $v$ may be increased if scans in the direction of decreasing potential are discarded.

Probing of the electric potential by SVM has been shown experimentally to be an inherently slow process. Reaching steady state between the semiconductor sample and measurement circuit must occur for accurate measurement of the local sample potential. First-order time constants of approaching steady state are on the order of $10^{-2} \mathrm{~s}$ (tracking increasing and decreasing potentials) on $n$-type InP, and $10^{-1} \mathrm{~s}$ (tracking increasing potential) and $1 \mathrm{~s}$ (tracking decreasing potential) on $p$-type InP. For accurate measurements, the SVM scan speed must be limited by the ratio of spatial resolution to the slowest time required to reach steady state.

${ }^{1}$ T. Trenkler, P. De Wolf, and W. Vandervorst, J. Vac. Sci. Technol. B 16, 367 (1998).

${ }^{2}$ T. Trenkler, R. Stephenson, P. Jansen, and W. Vandervorst, J. Vac. Sci. Technol. B 18, 586 (2000).

${ }^{3}$ D. Ban, E. H. Sargent, K. Hinzer, St. J. Dixon-Warren, A. J. SpringThorpe, and J. K. White, Appl. Phys. Lett. 82, 4166 (2003).

${ }^{4}$ D. Ban, E. H. Sargent, St. J. Dixon-Warren, G. Latel, A. J. SpringThorpe, G. Knight, and J. K. White, IEEE J. Quantum Electron. (to be published).

${ }^{5}$ V. V. Zavyalov, J. S. McMurray, S. D. Stirling, C. C. Williams, and H. Smith, J. Vac. Sci. Technol. B 18, 549 (2000).

${ }^{6}$ J. M. R. Weaver and D. W. Abraham, J. Vac. Sci. Technol. B 9, 1559 (1991).

${ }^{7}$ M. Nonnenmacher, M. P. O'Boyle, and H. K. Wickramasinghe, Appl. Phys. Lett. 58, 2921 (1991).

${ }^{8}$ D. Ban, E. H. Sargent, St. J. Dixon-Warren, I. Calder, T. Grevatt, G. Knight, and J. K. White, J. Vac. Sci. Technol. B 20, 2401 (2002).

${ }^{9}$ R. P. Lu, K. L. Kavanagh, S. Dixon-Warren, A. J. SpringThorpe, R. Streater, and I. Calder, J. Vac. Sci. Technol. B 20, 1682 (2002).

${ }^{10}$ T. Hantschel, P. Niedermann, T. Trenkler, and W. Vandervorst, Appl. Phys. Lett. 76, 1603 (2000).

${ }^{11}$ Keithley 6517A Electrometer/High Resistance Meter Datasheet (2002).

${ }^{12}$ A. G. Fitzgerald, Y. Fan, P. John, C. E. Troupe, and J. I. R. Wilson, J. Vac. Sci. Technol. B 18, 2714 (2000).

${ }^{13}$ S. Richter, M. Geva, J. P. Garno, and R. N. Kleiman, Appl. Phys. Lett. 77, 456 (2000).

${ }^{14}$ L. Dierderich, O. M. Küttel, P. Aebi, and L. Schlapbach, Surf. Sci. 418, 219 (1998).

${ }^{15}$ Handbook Series on Semiconductor Parameters, edited by M. Levinshtein, S. Rumyantsev, and M. Shur (World Scientific, New Jersey, 1996).

${ }^{16}$ D. A. Neamen, Semiconductor Physics and Devices: Basic Principles, 3rd ed. (McGraw-Hill, New York, 2003). 